\title{
Hipertensão Arterial Experimental e Prenhez em Ratas: Uso do Modelo Goldblatt I (1 rim - 1 clipe)
}

\author{
Experimental hypertension and pregnancy in rats: use of Goldblatt I \\ (one kidney - one clip model)
}

\author{
Rogério Dias ${ }^{1}$, José Carlos Souza Trindade ${ }^{2}$, \\ Marilza Vieira Cunha Rudge ${ }^{1}$, Paulo Roberto Curi ${ }^{3}$
}

\section{RESUMO}

\begin{abstract}
Objetivo: desenvolver o modelo experimental de hipertensão tipo Goldblatt I (1 rim - 1 clipe), em ratas, para estudar a interação entre hipertensão e prenhez.

Métodos: o experimento foi dividido em 5 periodos: adaptação (2 semanas), cirúrgico (1 semana), desenvolvimento da hipertensão (6 semanas), acasalamento e estabilização da pressão arterial (6 semanas) e prenhez (3 semanas). Foram utilizadas 82 ratas virgens da raça Wistar, pesando entre 180-240 gramas e com idade entre 3 e 4 meses. As ratas foram sorteadas para compor os 4 grupos experimentais (controle, manipulação, nefrectomia $e$ hipertensão) e estudadas em 15 momentos distintos $\left(\begin{array}{lll}M_{1} & \text { a } & M_{15}\end{array}\right)$. A hipertensão foi induzida experimentalmente pela técnica de Goldblatt I (1 rim, 1 clipe), que consiste na constrição da artéria renal esquerda e nefrectomia contralateral. Posteriormente, foram realizadas medidas periódicas da pressão arterial pelo método da pletismografia de cauda (PAC).

Resultados: os animais sem tratamento cirúrgico (controle) e com manipulação não apresentaram alterações na PAC durante o experimento. A nefrectomia determinou discreta elevação da PAC. Nos grupos de ratas prenhes, observou-se tendência a discreta diminuição da PAC, que se acentuou no final da prenhez.

Conclusões: o modelo experimental foi adequado para o objetivo de nosso estudo, pois permitiu a obtenção de animais hipertensos.
\end{abstract}

PALAVRAS-CHAVE: Hipertensão e gravidez. Complicações da gravidez. Modelos experimentais.

\section{Introdução}

A hipertensão arterial na gravidez é responsável por elevada morbidade materna e

\footnotetext{
${ }^{1}$ Departamento de Ginecologia e Obstetrícia, Faculdade de Medicina de Botucatu, UNESP

2 Departamento de Urologia, Faculdade de Medicina de Botucatu, UNESP

${ }^{3}$ Depto. de Estatística da Faculdade de Medicina de Botucatu, UNESP. Laboratório de Investigação Experimental do Departamento de Ginecologia e Obstetrícia, Faculdade de Medicina de Botucatu, UNESP.

Correspondência:

Rogério Dias

Departamento de Ginecologia e Obstetrícia

Faculdade de Medicina de Botucatu - UNESP

18618-000 - Botucatu - SP.

Este trabalho foi parcialmente financiado pelo CNPq- Processo: $n^{\circ} 300388 / 98-9$.
}

perinatal e pelo agravamento do prognóstico relacionado com o desenvolvimento físico e neuropsicomotor do recém-nascido ${ }^{21}$. Associa-se com aumento da incidência da insuficiência placentária, tendo como conseqüência o retardo de crescimento intra-uterino e a depressão neonatal, principalmente quando não se controla a hipertensão na gestação $0^{11,22}$.

De acordo com a Sociedade Internacional para Estudo de Hipertensão na Gestação, a hipertensão é diagnosticada quando a pressão arterial diastólica (PAD) é de $110 \mathrm{mmHg}$ ou mais em qualquer ocasião ou quando há PAD de 90 $\mathrm{mmHg}$ ou mais, em duas ocasiões consecutivas, com intervalo de 4 horas $^{8}$.

Para animais de experimentação, a literatura considera hipertensão quando os niveis pressóricos 
são iguais ou superiores a $130 \mathrm{mmHg}$ em duas medidas sucessivas ${ }^{13}$. Vários métodos têm sido empregados para a produção experimental de hipertensão no rato, como as várias técnicas de Goldblatt: I (GI) (1 rim - 1 clipe), caracterizada pela constrição da artéria renal esquerda e nefrectomia contralateral; II (GII) (2 rins - 1 clipe), ambos os rins permanecem intactos, mas há constricção da artéria renal de um deles; III (GIII) (2 rins - 2 clipes), com redução dos diâmetros de ambas artérias renais. A técnica de produção de hipertensão renovascular tipo Goldblatt I (GI), modelo 1 rim - 1 clipe, foi descrita por Goldblatt et al. ${ }^{12}$ para cães e, mais tarde, adaptada por Schaffenburg ${ }^{19}$ para animais de pequeno porte.

O mecanismo básico de hipertensão induzida no modelo Goldblatt I é a expansão de volume corporal com aumento da pressão circulatória média, volume sangüineo e rendimento cardíaco, caracterizando modelo hipertensivo com adaptação adequada do organismo materno ${ }^{23}$. No modelo Golblatt II (GII) (2 rins - 1 clipe), o sistema renina-angiotensina é o principal fator no desenvolvimento da hipertensão ${ }^{10}$.

Considerando-se a importância dos efeitos da hipertensão arterial sobre a prenhez, tivemos como objetivos neste trabalho a montagem do modelo experimental Goldblatt I ( 1 rim - 1 clipe) para o estudo da hipertensão arterial na prenhez e a avaliação do comportamento dos níveis pressóricos maternos durante a prenhez.

\section{Material e Métodos}

Foram utilizadas 82 ratas virgens da raça Wistar, pesando entre 180 e 240 gramas, com idade entre 3 e 4 meses. A seqüência experimental de 18 semanas foi dividida em cinco períodos: adaptação, cirúrgico, desenvolvimento da hipertensão, acasalamento e prenhez (Figura 1).

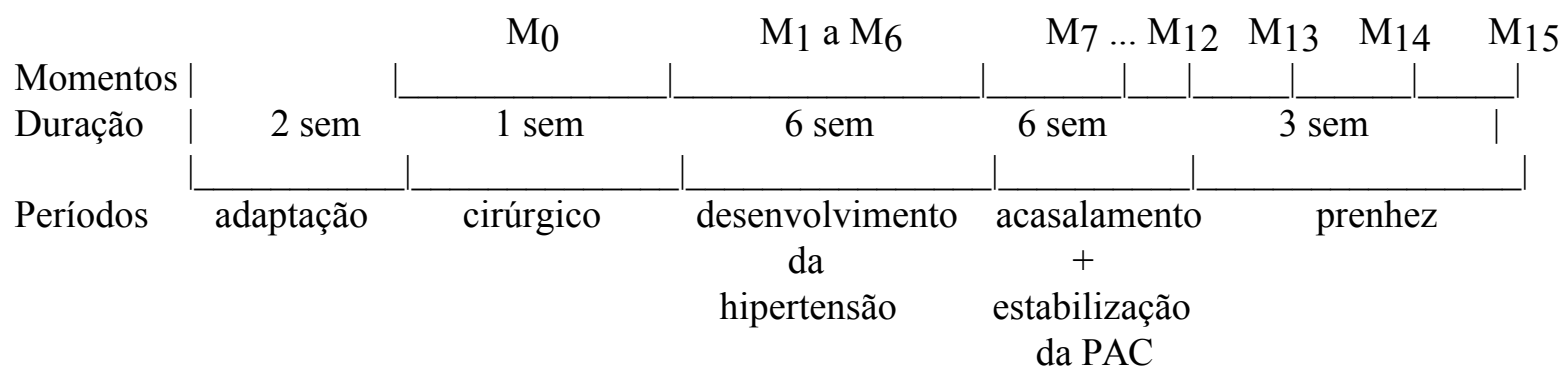

Figura 1 - Seqüência experimental para desenvolvimento de hipertensão associada à gravidez em ratas.

O período de adaptação durou 15 dias e os animais permaneceram em gaiolas individuais com temperatura ambiente entre $22^{\circ}$ e $25^{\circ} \mathrm{C}$, com luz ambiente, recebendo água e ração "ad libitum". Neste período foi determinada a pressão arterial da cauda (PAC) e foram excluídas as ratas com níveis pressóricos iguais ou superiores a $130 \mathrm{mmHg}$ $\left(\mathrm{M}_{0}\right)$. Após este periodo, as ratas foram distribuídas por sorteio para os quatro grandes grupos experimentais: controle (1), manipulação (2), nefrectomia (3) e hipertenso (4).

No período cirúrgico as ratas dos grupos 2, 3 e 4 foram anestesiadas com éter sulfúrico, superficial o suficiente para manipulação indolor dos animais. A seguir, depois de fixadas em posição supina, foi realizada tricotomia da região do abdome e anti-sepsia da pele com álcool iodado. Após a laparotomia mediana, as ratas do grupo 2 foram submetidas à abertura das lojas renais e dissecção dos vasos de ambos os pedículos. As do grupo 3 foram submetidas à nefrectomia direita e dissecção da artéria renal esquerda. As do grupo 4 a nefrectomia direita e estenose da artéria renal esquerda com clipe de prata de 8 × $2 \mathrm{~mm}$ de abertura ajustável. O grau de constrição arterial foi proporcional ao peso do animal ${ }^{14}$.

A incisão foi fechada com fio de algodão 00 em 2 planos e os animais receberam $0,1 \mathrm{ml}$ da associação penicilina-estreptomicina no pósoperatório imediato, na primeira e na segunda semana após a cirurgia.

No período de desenvolvimento da hipertensão $\left(\mathrm{M}_{1}\right.$ a $\left.\mathrm{M}_{6}\right)$, a PAC foi determinada 2 vezes por semana com o pletismógrafo de cauda, aquecendo-se previamente o animal em ambiente entre $40^{\circ}$ e $45^{\circ} \mathrm{C}$ durante 5 minutos. Foram considerados hipertensos todos os animais que apresentaram níveis pressóricos iguais ou superiores a $130 \mathrm{mmHg}$, em duas medidas sucessivas $^{14}$.

A seguir, as ratas foram sorteadas para compor os subgrupos prenhes $(\mathrm{P})$ e nãoprenhes (NP), constituindo-se desta forma oito grupos experimentais: quatro prenhes e 
quatro não-prenhes (Tabela 1).

Tabela 1 - Grupos experimentais e respectivas denominações

\begin{tabular}{clcc}
\hline Grupos & Denominação & Prenhe & Não-Prenhe \\
\hline 1 & Controle & P-1 & NP-1 \\
2 & Manipulação & P-2 & NP-2 \\
3 & Nefrectomia direita & P-3 & NP-3 \\
4 & Hipertenso & P-4 & NP-4 \\
\hline
\end{tabular}

No período de acasalamento as ratas dos grupos prenhes foram acasaladas e a prenhez diagnosticada pela análise microscópica do esfregaço vaginal. A presença de espermatozóide caracterizou o "dia zero" da prenhez ${ }^{6}$. Durante esse período, as ratas dos grupos não-prenhes permaneceram em gaiolas coletivas, à semelhança dos animais em acasalamento, porém sem o macho. No periodo de prenhez, os animais permaneceram em gaiolas individuais, à semelhança dos animais não-prenhes.

Os animais dos 8 grupos experimentais foram observados quanto aos niveis pressóricos em 15 momentos (M) distintos, previamente delineados: M0: pré-cirúrgico; $M_{1}$ a $M_{6}$ : correspondentes as 6 semanas para o desenvolvimento da hipertensão; $\mathrm{M}_{7}$ a $\mathrm{M}_{11}$ : nas 5 semanas para o acasalamento e estabilização da PAC; $\mathrm{M}_{12}$ : dia pré-gravídico e $\mathrm{M}_{13}$, $\mathrm{M}_{14}$ e $\mathrm{M}_{15}$ correspondentes às 3 semanas de prenhez.

Utilizamos análise de perfil ${ }^{7}$ para o referido estudo proposto. As estatísticas calculadas em cada hipótese testada tiveram suas significâncias verificadas, confrontando-as com os respectivos valores críticos para $\mathrm{p}=0,05$.

\section{Resultados}

No início do experimento, do ponto de vista clínico, todos os animais estavam livres de doenças. A antibioticoterapia profilática foi eficiente na proteção contra infecções, com adequada cicatrização da ferida operatória. As ratas evoluíram bem durante a prenhez, sem nenhum sinal de infecção evidente por ocasião do sacrificio dos animais.

A evolução da pressão arterial, acompanhada pela pletismografia de cauda, está representada nas Figuras 2 e 3 . Os animais hipertensos se mantiveram com niveis pressóricos acima de 130 $\mathrm{mmHg}$, tendo sido posteriormente sorteados e acasalados.

Para os animais sem tratamento cirúrgico

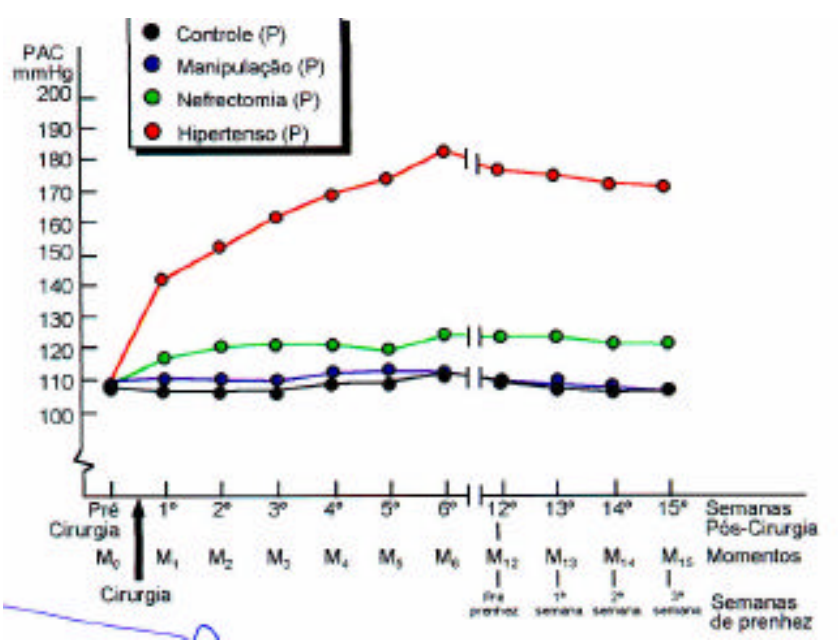

Figura 2 - Médias $(\bar{X})$ dos valores da pressão arterial da cauda (PAC) em $\mathrm{mmHg}$ das ratas dos 4 grupos experimentais prenhes $(P)$ : controle $(P)$; manipulação $(P)$; nefrectomia $(P)$; e hipertens+São $(P)$, observados em todos os momentos do experimento $\left(M_{0}\right.$ a $\left.M_{15}\right)$

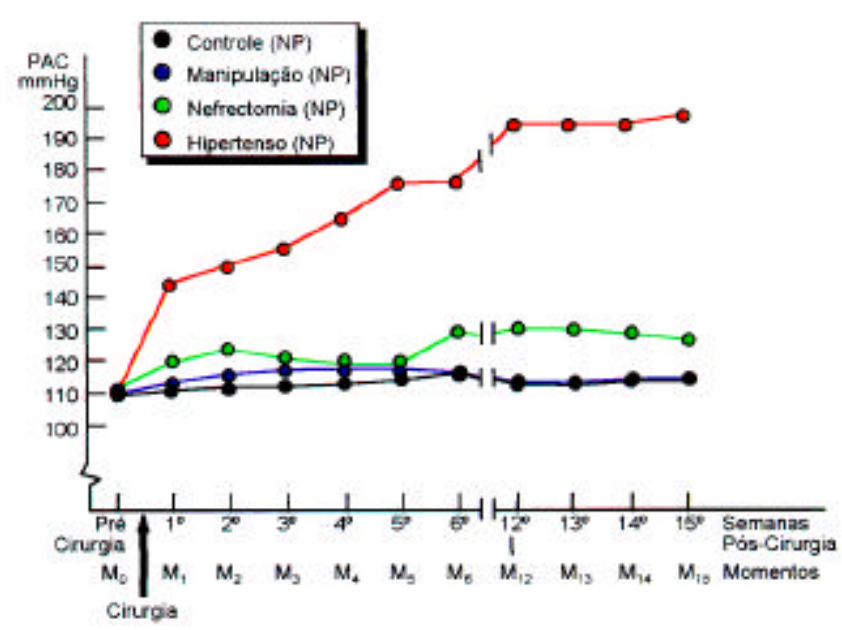

Figura 3 - Médias $(\bar{x})$ dos valores da pressão arterial da cauda (PAC) em mmHg das ratas dos 4 grupos experimentais não-prenhes (NP): controle (NP); manipulação (NP); nefrectomia (NP); e hipertensão (NP) observados em todos os momentos do experimento $\left(\mathrm{M}_{0}\right.$ a $\left.\mathrm{M}_{15}\right)$.

(grupo controle) e com manipulação cirúrgica (grupo manipulação), a média dos valores observados em cada momento permaneceu inalterada durante todo o transcurso do experimento. Isto se explica pelo fato de o primeiro grupo não ter sofrido nenhum tratamento experimental e o segundo, apenas a manipulação dos vasos do pedículo renal.

A nefrectomia direita determinou discreta elevação da pressão arterial, que não significou hipertensão, pois os niveis pressóricos foram inferiores a $130 \mathrm{mmHg}$.

Nos grupos de ratas prenhes, observa-se tendência a discreta diminuição da pressão arterial, embora sem diferença significativa, quando 
comparamos os momentos pré-prenhez $\left(\mathrm{M}_{12}\right)$ e os durante a prenhez $\left(\mathrm{M}_{13}, \mathrm{M}_{14}\right.$ e $\left.\mathrm{M}_{15}\right)$ (Figura 2).

Há diminuição significativa da pressão arterial em $\mathrm{M}_{15}$ comparada com o valor pré-prenhez $\left(\mathrm{M}_{12}\right)$ para os grupos prenhes (Figura 2). Esta diminuição da pressão arterial na prenhez simula o observado em humanos e em animais ${ }^{16}$ e também o observado por Goldblatt et al. ${ }^{12}$ em cadelas prenhes.

A análise estatística nos mostra que entre os resultados apresentados na Figura 2 (valores da PAC das ratas dos 4 grupos experimentais prenhes - P) e os da Figura 3 (valores da PAC das ratas dos 4 grupos experimentais não-prenhes NP) há diferenças significativas.

\section{Discussão}

Vários são os animais utilizados em modelos experimentais para o estudo da hipertensão durante a prenhez. Dentre todos, o mais empregado é o rato, pois reúne várias condições favoráveis, como: baixo custo, fácil obtenção na fase reprodutiva, facilidade do diagnóstico da prenhez, curta duração do periodo gestacional, facilidade na medida periódica da pressão arterial da cauda com o animal acordado, entre outras ${ }^{13,17,24}$.

Como pudemos observar, o uso do modelo Goldblatt I torna hipertensos todos os animais. Para um melhor entendimento, fizemos uma correlação na qual cada semana de prenhez da rata foi considerada equivalente a cada trimestre da gestação humana ${ }^{15}$. Para melhor padronizarmos nossa metodologia, todas as ratas usadas no experimento eram virgens, a fim de descartarmos variações das respostas em decorrência de prenhez anterior. Como a duração do experimento foi extensa, os animais foram mantidos em condições controladas de temperatura e luz, a fim de minimizar os efeitos sazonais sobre o metabolismo da rata prenhe.

A literatura tem mostrado a importância do grau de constricção do clipe na artéria em relação ao crescimento do animal ao longo da experiência e por esse motivo obedeceu-se à relação peso/grau de estreitamento ${ }^{14,20}$. As ratas estudadas desenvolveram hipertensão após a constrição arterial e nefrectomia contralateral, determinandose com precisão o início da elevação da pressão arterial. Este fato permitiu identificar os animais que se mantiveram hipertensos, reproduzindo, de maneira adequada, o modelo proposto.

Para a medida da pressão arterial da rata, utilizou-se o método pletismógrafo de manguito de cauda ("tail-cuff method"), por ser amplamente empregado, depender de técnica simples, ser rápido e não necessitar do uso de anestésico ${ }^{9}$.

A pressão arterial da cauda (PAC) foi semelhante para todos os grupos experimentais no início do estudo, indicando a homogeneidade das amostras.

A hipertensão arterial modelo Goldblatt I (1 rim - 1 clipe) foi por nós reproduzida de maneira adequada, conforme trabalhos da literatura ${ }^{18,20}$.

Existem vários trabalhos nos quais se discute a influência da prenhez na hipertensão renal experimental em ratas, e nos quais se tenta explicar sua etiologia. Uma das hipóteses seria o aumento na produção de progesterona, que é um antagonista da atividade mineralocorticóide (aldosterona) e importante fator natriurético. Sugere-se também que alterações endócrinas produziriam redução na resistência periférica com relaxamento de arteriolas, induzida pela progesterona ou pelas prostaglandinas estimuladas pelo estrogênio. A gestação está associada ao aumento na produção de prostaglandina (PGE2), cininas e outros fatores humorais, que influenciariam o metabolismo de sódio e água. É possivel que a rata prenhe hipertensa, por razões desconhecidas, possa ser mais sensivel a tais fatores $^{2,16}$

Ahokas et al. ${ }^{1}$ sugerem que a queda da pressão arterial durante a prenhez estaria relacionada às alterações hemodinâmicas (dilatação do leito vascular devido ao aumento da circulação uterino-placentária) decorrentes do desenvolvimento da placenta.

Admite-se que esta diminuição de pressão arterial é decorrência de fenômenos gestacionais, em que a placenta funcionaria como um shunt artério-venoso ${ }^{4,5}$. As placentas da rata, da coelha e da mulher, embora apresentem diferenças na estrutura vascular, podem constituir anastomoses artério-venosas de baixa resistência. Estas, por sua vez, diminuem a resistência periférica e seriam responsáveis pela queda da pressão arterial durante a gestação.

Segundo outros autores, a queda da pressão arterial sangüínea na gestação normal é devida à vasodilatação periférica, principal fator responsável pela diminuição da resistência vascular sistêmica, mas esse mecanismo não é conhecido de forma adequada $^{21}$.

Zamorano et al. ${ }^{25}$ e Belizàn et al. ${ }^{3}$ observaram queda da pressão arterial sangüínea em ratas hipertensas quando havia privação de cálcio nos últimos dias de gestação. Tal fato é atribuído a um 
aumento na produção de prostaglandinas da série PGE, que tem efeito vasodilatador.

As observações de Belizàn et al. ${ }^{3}$ a respeito do comportamento dos níveis pressóricos em ratas hipertensas são semelhantes às verificadas por nós, mesmo trabalhando com modelos experimentais diferentes.

A comparação dos grupos prenhes entre si (Figura 2) mostra que os grupos prenhes, controle e manipulação cirúrgica, mantêm os mesmos niveis pressóricos. O grupo submetido apenas a nefrectomia apresenta niveis pressóricos intermediários entre a normalidade e a hipertensão.

A análise estatística desses resultados evidencia que as diferenças entre grupos são significativas em todos os momentos do período gestacional estudados. A observação desses resultados está de acordo com o esperado, uma vez que nos dois primeiros grupos não houve interferência na circulação renal, ao passo que, nos dois últimos, o tratamento utilizado determinou diminuição da massa renal do grupo da nefrectomia (P) e diminuição da massa renal e do fluxo sangüíneo renal no grupo da hipertensão (hipertenso P). Esses dois últimos fatores associados devem estar relacionados com a etiopatogenia da hipertensão neste grupo experimental.

A comparação entre os quatro grupos prenhes $(\mathrm{P})$ com os seus respectivos grupos não prenhes (NP) no periodo de prenhez $\left(\mathrm{M}_{13}, \mathrm{M}_{14}\right.$ e $\left.\mathrm{M}_{15}\right)$ mostra que todos os grupos apresentaram queda dos niveis pressóricos. Entretanto, nos grupos nãoprenhes, a pressão arterial manteve-se estacionária. Esses achados levam-nos a concluir que a prenhez diminui os niveis pressóricos das ratas.

Nas condições de nosso trabalho e com base nos resultados obtidos, podemos concluir que a técnica de Goldblatt I, utilizada para produzir hipertensão em ratas, foi eficaz, pois permitiu a obtenção de animais hipertensos, confirmando o achado de outros autores, e que a prenhez determinou uma queda dos niveis pressóricos nos seus momentos finais.

\section{SUMMARY}

Purpose: to develop an experimental model in rats to study the interaction between hypertension and pregnancy.

Methods: the present experiment was divided into 5 periods: adaptation (2 weeks), surgical procedures (1 week), hypertension development (6 weeks), mating and blood pressure stabilization (6 weeks), and gestational period (3 weeks). A total of 82 animals in reproductive age, weighing from 180 to $240 \mathrm{~g}$, were used. They were randomly assigned to the 4 different groups (control, handled, nephrectomy and hypertension) and renal hypertension was produced by a controlled constriction of the main left renal artery, according to the technique described by Goldblatt, and contralateral nephrectomy (Goldblatt I - one kidney, one clip hypertension). They were studied at 15 precise moments. Afterwards, periodic blood pressure determinations were made by the tail plethysmographic method.

Results: pregnancy caused a fall in blood pressure levels in the rat.

Conclusion: the experimental model was adequate for the purposes of the study, since it proved to be efficient in producing hypertension.

KEY WORDS: Hypertension and pregnancy. Complications in pregnancy. Experimental hypertension in rats Experimental models.

\section{Referências}

1. Ahokas RA, Reynolds SL, Anderson GD, Lipshitz J. Uteroplacental blood flow in the hypertensive, term pregnant, spontaneously hypertensive rat. Am J Obstet Gynecol 1987; 156:1010-5.

2. Aoi W, Gable D, Cleary RE, Young PC, Weinberger $\mathrm{MH}$. The antihypertensive effect of pregnancy in spontaneously hypertensive rats. Proc Soc Exp Biol 1976; 153:13-15.

3. Belizàn JM, Pineda O, Sainz E, Menendez LA, Villar J. Rise of blood pressure in calcium-deprived pregnant rats. Am J Obstet Gynecol 1981; 141:163-9.

4. Bernheim J. Hypertension in pregnancy. Nephron 1997; 76: 254-63.

5. Burwell CS. The placenta as a modified arteriovenous fistula, considered in relation to the circulatory adjustments of pregnancy. Am J Med Sci 1938; 195:1-7.

6. Camano L. Contribuição para o estudo histoquímico do muco do epitélio vaginal da rata (Rattus norvegicus var. albinus, Rodentia, Mammalia), no ciclo estral, na prenhez e na pós-parturição. Tese (Doutorado) São Paulo: Escola Paulista de Medicina, 1968.

7. Curi PR. Análise de medidas repetidas em experimentos biológicos. Rev Bras Estat 1980; 41:137-50.

8. Davey DA, MacGillivray I. The classification and definition of hypertensive disorders. Clin Exp Hypertens B 1986; 5:97-133.

9. Dias R. Repercussões da hipertensão arterial renovascular sobre a prenhez e os recém-nascidos. Estudo experimental em ratos. Dissertação (Mestrado). Botucatu: Faculdade de Medicina de Botucatu, 1984. 
10.Ferrario CM, Carretero OA. Hemodynamics of experimental renal hypertension. In: Jong, W, editor. Handbook of Hypertension: Experimental and Genetic Models of Hypertension. New York: Elsevier; 1984. v.4, p.54-80.

11.Gant NF, Worley RJ, Cunningham FC, Whalley PJ. Clinical management of pregnancy-induced hypertension. Clin Obstet Gynecol 1978; 21:397-409.

12.Goldblatt H, Kahn JR, Hanzal RF. Studies on experimental hypertension. The effect on blood pressure of constriction of the abdominal aorta above and below the site of origin of both main renal arteries. J Exp Med 1939; 69:649-74.

13.Karlsson K, Ljungblad U, Lundgren Y. Blood flow of the reproductive system in renal hypertensive rats during pregnancy. Am J Obstet Gynecol $1982 ; 142: 1039-44$.

14.Krieger EM. Neurogenic hypertension in the rat. Circ Res 1964; 15:511-21.

15.Ling PR, Bistrian BR, Blackburn GL, Istfan N. Effect of fetal growth on maternal protein metabolism in postabsorptive rat. Am J Physiol 1987; 252:E380-90.

16.Lorenz RP, Picchio LP, Weisz J, Lloyd T. The relationship between reproductive perfomance and blood pressure in the spontaneously hypertensive rat. Am J Obstet Gynecol 1984; 150:519-23.

17.Nitzan M, Orloff S, Chrzanowaka BL, Schulman JD. Intrauterine growth retardation in renal insufficiency: An experimental model in the rat. Am J Obstet Gynecol 1979; 133:40-3.
18. Ribeiro AB. Contribuição para o estudo da gênese da hipertensão no modelo Goldblatt I em ratos. Tese (Doutorado) São Paulo: Escola Paulista de Medicina, 1977.

19.Schaffenburg CA. Device to control constriction of main renal artery for production of hypertension in small animals. Proc Soc Exp Biol Med 1959; 101:676-7.

20.Sumitani M. Estudo da readaptação dos pressorreceptores na hipertensão de longa duração. Tese (Mestrado). Ribeirão Preto: Faculdade de Medicina de Ribeirão Preto, Universidade de São Paulo, 1981.

21.Svensson A. Hypertension in pregnancy long-term effects on blood pressure in mothers and children. Acta Med Scand (Suppl) 1985; 695:1-50.

22.Visser GHA, Huisman A, Saathof PWF, Sinnige HA. Early fetal growth retardation: obstetric background and recurrence rate. Obstet Gynecol 1986; 67:40-3.

23.Wallenburg H.C.S. Prevention of hypertensive disorders in pregnancy. Clin Exp Hypertens 1988; 37:121-37.

24.Wigglesworth JS. Experimental growth retardation in foetal rat. J Pathol Bacteriol 1964; 88:1-13.

25.Zamorano B, Terragno A, McGiff JC, Terragno NA. A prostaglandin mechanism may contribute to the regulation of blood pressure in spontaneously hypertensive rats during pregnancy. Adv Prostaglandin Thromboxane Res 1980; 7:807-10.

\section{$\mathrm{RBGO}$}

\section{é uma publicação da FEBRASGO}

e que aceita artigos provenientes de sinecologistas, obstetras e de outras especialidades.

\section{Portanto, publique!!!}

\section{Mande já seu artigo para RBGO}

